\title{
A THEOREM ON THE CLUSTER SETS OF PSEUDO-ANALYTIC FUNCTIONS
}

\author{
KIYOSHI NOSHIRO
}

1. Let $D$ be an arbitrary connected domain and $w=f(z)=u(x, y)+i v(x, y)$, $z=x+i y$, be an interior transformation in the sense of Stoilow in $D$. Denote by $r$ a set, in $D$, such that $D$ and the derived set $\gamma^{\prime}$ of $\gamma$ have no point in common. We suppose that

$$
\begin{gathered}
u_{x}, u_{y}, v_{x}, v_{y} \text { exist and are continuous in } D^{*}=D-\gamma ; \\
J(z)=\left|\begin{array}{ll}
u_{x} & u_{y} \\
v_{x} & v_{y}
\end{array}\right|>0 \text { at every point in } D^{*} ;
\end{gathered}
$$

(iii) the function $q(z)$ defined as the ratio of the major and minor axes of an infinitesimal ellipse with centre $f(z)$, into which an infinitesimal circle with centre at each point $z$ of $D^{*}$ is transfomed by $w=f(z)$, is bounded in $D^{*}: q(z) \leqq A$.

$f(z)$ is then called pseudo-meromorphic $(A)$ in $D .{ }^{1)}$

Next, suppose that $w=f(z)$ is pseudo-meromorphic $(A)$ in $D$. Let $C$ be the boundary of $D, E$ be a closed set of capacity ${ }^{2}$ zero, included in $C$, and $z_{0}$ be a point in $E$. We can associate with $z_{0}$ three cluster sets $S_{z_{0}}^{(D)}, S_{z_{0}}^{(C)}$ and $S_{z_{0}}^{*(C)}$ as follows: $S_{z_{0}}^{(D)}$ is the set of all values $\alpha$ such that $\lim _{\nu \rightarrow \infty} f\left(z_{\nu}\right)=\alpha$ with a sequence $\left\{z_{\nu}\right\}$ of points tending to $z_{0}$ inside $D . S_{z_{0}}^{*(c)}$ is the intersection $\bigcap_{r} M_{r}$, where $M_{r}$ denotes the closure of the union $\bigcup_{\zeta^{\prime}} S_{\zeta^{\prime}}^{(D)}$ for all $\zeta^{\prime}$ belonging to the common part of $C-E$ and $U\left(z_{0}, r\right):\left|z-z_{0}\right|<r^{\prime}$. In the particular case when $E$ consists of a single point $z_{0}$, we denote $S_{z_{0}}^{*(C)}$ by $S_{z_{0}}^{(C)}$ for simplicity. Obviously $S_{z_{0}}^{(D)}$ and $S_{z_{0}}^{*(C)}$ are closed sets such that $S_{z_{0}}^{*(C)} \subset S_{z_{0}}^{(D)}$ and $S_{z_{0}}^{(D)}$ is always non-empty while $S_{z_{0}}^{*(r)}$ becomes empty if and only if there exists a positive number $r$ such that $C-E$ and $U\left(z_{0}, r\right)$ have no point in common.

In the particular case where $w=f(z)$ is single-valued meromorphic in $D$, the following theorems concerning the cluster sets $S_{z_{0}}^{(D)}, S_{z_{0}}^{(C)}$ and $S_{z_{0}}^{*(C)}$ are known:

Received February 20, 1950.

1) For the definition of pseudo-meromorphic functions, Cf. S. Kakutani: Applications to the theory of pseudo-regular functions to the type-problem of Riemann surfaces, Jap. Journ. of Math. Vol. 13 (1937), pp. 375-392. R. Nevanlinna: Eindeutige analytische Funktionen, Berlin, 1936, p. 343.

2) "Capacity" means logarithmic capacity in this note. 
Theorem I. (Iversen-Beurling-Kunugui) ${ }^{3)} \quad B\left(S_{z_{0}}^{(D)}\right) \subset S_{z_{0}}^{(C)}$, where $B\left(S_{z_{0}}^{(D)}\right)$ denotes the boundary of $S_{z_{0}}^{(D)}$, or what is the same, $\boldsymbol{Q}=S_{z_{0}}^{(D)}-S_{z_{0}}^{(C)}$ is an open set.

Theorem II. (Beurling-Kunugui) ${ }^{4)}$ Suppose that $\Omega=S_{z_{0}}^{(D)}-S_{z_{0}}^{(C)}$ is not empty and denote by $\Omega_{n}$ any component of $\Omega$. Then $w=f(z)$ takes every value, with two possible exceptions, belonging to $\Omega_{n}$ infinitely often in any neighbourhood of $z_{0}$.

Theorem I*. (Tsuji) ${ }^{5)} B\left(S_{z_{0}}^{(D)}\right) \subset S_{z_{0}}^{*(C)}$, that is, $\Omega=S_{z_{0}}^{(D)}-S_{z_{0}}^{*(C)}$ is an open set.

Theorem II*. (Kametani-Tsuji) ${ }^{6)}$ Suppose that $\Omega=S_{z_{0}}^{(D)}-S_{z_{0}}^{*(c)}$ is not empty. Then $w=f(z)$ takes every value, except a possible set of $w$-values of capacity zero, belonging to $\Omega$ infinitely often in any neighbourhood of $z_{0}$.

The object of the present note is to propose the following

THEOREM 1. Suppose that $E$ is included in a single boundary-component $C_{0}$ of $C$ and $w=f(z)$ is pseudo-meromorphic $(A)$ in $D$. Then $\Omega=S_{z_{0}}^{(D)}-S_{z_{0}}^{*(C)}$ is an open set. Suppose further that $\Omega$ is not empty. Then $w=f(z)$ takes every value, with two possible exceptions, belonging to any component $\Omega_{n}$ of $\Omega$ infinitely often in any neighbourhood of $z_{0}$.

Remark. It is obvious that Theorem 1 contains Theorems I and II ${ }^{\text {) }}$ and holds good provided that $D$ is simply connected.) There is an anticipation that Theorems I* and II* may be probably true when $w=f(z)$ be pseudo-meromor-

3) F. Iversen: Sur quelques propriétés des fonctions monogènes au voisinage d'un point singulier, Öfv. af Einska Vet-Soc. Förh. 58 (1916).

K. Kunugui: Sur un théorème de M. M. Seidel-Beurling, Proc. Acad. Tokyo, 15 (1939); Sur un problème de M. A. Beurling, Proc, Acad. Tokyo, 16 (1940); Sur l'allure d'une fonction analytique uniform au voisinage d'un point frontière de son domaine de définition, Jap. Journ. of Math. 18 (1942), pp. 1-39.

A. Beurling: Études sur un problème de majoration, Thèse de Upsal, 1933; Cf. pp. 100-103.

4) Beurling: 1. c. 3); Kunugui: 1. c. 3).

5) M. Tsuji: On the cluster set of a meromorphic function, Proc. Acad. Tokyo, 19 (1943); On the Riemann surface of an inverse function of a meromorphic function in the neighbourhood of a closed set of capacity zero, Proc. Acad. Tokyo, 19 (1943).

6) Tsuji: 1. c. 5). . S. Kametani: The exceptional values of functions with the set of capacity zero of essential singularities, Proc. Acad. Tokyo, 17 (1941), pp. 429-433.

7) Recently E. Sakai has obtained some interesting results concerning pseudo-meromorphic functions. Theorem 1 answers affirmatively a problem represented by him. Cf. E. Sakai: Note on pseudo-analytic functions, forthcoming Proc. Acad. Tokyo.

8) The special case where $D$ is simply connected and $w=f(z)$ is single-valued meromorphic in $D$ has been treated by the writer in another note. Cf. K. Noshiro: Note on the cluster sets of analytic functions, forthcoming Journ. Math. Soc. Japan. 
phic $(A)$ in $(D)$. But the writer has not yet succeeded in proving it.

2. To prove Theorem 1 we use two lemmas.

Lemma 1. Let $w=f(z)$ be pseudo-regular $(A)$ in a bounded domain $D$ and $E$ be a closed set of capacity zero, included in the boundary $C$ of $D$. If

$$
\varlimsup_{z \rightarrow \zeta}|f(z)| \leqq M
$$

for every point $\zeta$ of $C-E$ and $f(z)$ is bounded in a neighbourhood of every point $\zeta$ of $E$, then $|f(z)| \leqq M$ for all points $z$ in $D$.

Proof. We suppose, contrary to the assertion, that there exists a point $z_{0}$ in $D$ such that $\left|f\left(z_{0}\right)\right|>M$. Let $\Phi$ be the Riemannian image of $D$ by $w=f(z)$ and denote by $P_{0}$ the point on $\emptyset$ which corresponds to $z_{0}$. Consider the star-region $\boldsymbol{H}$ in Gross' sense formed by the sum of segments from $P_{0}$ with projection $w_{0}$ $=f\left(z_{0}\right)$ to singular points along all rays: $\arg \left(w-w_{0}\right)=\varphi$ on $\Phi$, whose projections lie in the half-plane $\Re\left[e^{-i \text { arg } w_{0}} \cdot\left(w-w_{0}\right)\right]>0$. We shall show that the linear measure of the set $\Gamma$ of arguments $\varphi$ of singular rays (by which we understand rays meeting singular points in finite distances) is equal to zero. Denote by $H_{R}$ the common part of $H$ and a circular disc $\left|w-w_{0}\right|<R$ and by $\Delta_{R}$ the image of $H_{R}$ by the inverse transformation of $w=f(z)$. Then, $A_{k}$ is a simply connected domain included in $D$. Since $E$ is a closed set of capacity zero, Evans' theorem ${ }^{9)}$ shows that there exists a distribution of positive mass $d \mu(a)$ entirely on $E$ such that

$$
u(z)=\int_{E} \log \left|\frac{1}{z-a}\right| d \mu(a), \mu(E)=1
$$

is harmonic outside $E$, excluding $z=\infty$, and has boundary value $+\infty$ at any point of $E$. Let $v(z)$ be its conjugate harmonic function and put

$$
t=\chi(z)=e^{u(z)+i v(z)}=\rho(z) e^{i v(z)} .
$$

For the sake of convenience, we call the function $t=\chi(z)$ "Evans" function." Let $C_{\lambda}$ be the niveau curve : $\rho(z)=$ const. $=\lambda(0<\lambda<+\infty)$. Then $C_{\lambda}$ consists of a finite number of simple closed curves surrounding $E$. Further, Evans' function has the property

$$
\int_{c_{\lambda}} d v(z)=\int_{C_{\lambda}} \frac{\partial u}{\partial n} d s=2 \pi,
$$

where $s$ denotes the arc length of $C_{\lambda}$ and $n$ is the inner normal of $C_{\lambda}$. Now

9) G. C. Evans: Potentials and positively infinite singularities of harmonic functions, Monatsheft für Math. und Phys. 43 (1935), pp. 419-424.

K. Noshiro: Contributions to the theory of the singularities of analytic functicns, Jap. Journ. of Math. 19 (1948), pp. 299-327. 
we consider the Riemannian image $\tilde{\Delta_{R}}$ of $\Delta_{R}$ by $t=\chi(z)$ and the function $w$ $=W(t)=f[z(t)]$ defined on $\tilde{\Delta}_{R}$. Let $\tilde{\Theta}_{\lambda}$ be the set of cross-cuts of $\tilde{\Delta}_{R}$ above the circle $|t|=\lambda$. We denote by $\lambda \theta(\lambda)$ the total length of $\widetilde{\Theta}_{\lambda}$ and $L(\lambda)$ that of the image of $\tilde{\Theta}_{\lambda}$ by $w=W(t)$. Then, applying a well-known method in proving Gross' theorem, we get

(4) $\int_{\lambda_{0}}^{\lambda} \frac{[L(\lambda)]^{2}}{\lambda \theta(\lambda)} d \lambda \leqq\left(A+\sqrt{\left.A^{2}-1\right)} \int_{\lambda_{0}}^{\lambda} \int_{\widetilde{\Theta}_{\lambda}} J(t) \lambda d \lambda d \theta \leqq \pi A R^{2}, \quad\left(0<\lambda_{0} \leqq \lambda\right)\right.$.

Since $\theta(\lambda) \leqq 2 \pi$, we have

$$
\lim _{\lambda \rightarrow \infty} L(\lambda)=0 .
$$

Accordingly, we see that the set $\Gamma$ of arguments $\varphi$ of singular rays is of linear measure zero. Consequently there exists at least one asymptotic path $\Lambda$ inside $D$ reaching a point $\zeta$ in $E$, along which $w=f(z)$ converges to $\infty$ as $z$ tends to 6. But this is a contradiction, since $f(z)$ is bounded in a neighbourhood of $\zeta$.

Remark. Lemma 1 is an immediate consequence from R. Nevanlinna's theorem ${ }^{10)}$ in the case when $w=f(z)$ is single-valued regular in $D$.

By a similar argument as in Lemma 1, we obtain, without difficulty,

Lemma 2. (An extension of Iversen's theorem) ${ }^{\text {in) }}$ Let $D$ be an arbitrary domain, $C$ being its boundary, and let $E$ be a closed set of capacity zero included in C. Suppose that $f(z)$ is pseudo-meromorphic $(A)$ in $D$ and $S_{z_{0}}^{(D)}-S_{z_{0}}^{*(C)}$ is not empty. If $w=f(z)$ does not take a value $\alpha$, contained in $S_{z_{0}}^{(D)}-S_{z_{0}}^{*(C)}$, infinitely often, then $\alpha$ is either an asymptotic value of $w=f(z)$ at $z_{0}$ or there is a sequence of accessible boundary points $\zeta_{n}$ in $E$ tending to $z_{0}$ such that $\alpha$ is an asymptotic. value at each $\zeta_{n}$.

3. Proof to Theorem 1. Let $w_{0}$ be an arbitrary value belonging to $S_{z_{0}}^{(D)}$ $-S_{z_{0}}^{*(c)}$. By hypothesis, there exists a circle $K:\left|z-z_{0}\right|=r$, arbitrarily small, such that $K \cdot E=0$ and $f(z) \neq w_{0}$ on $K \cdot D$. We may suppose that $w_{0}$ does not belong to the closure $M_{r}$ of the union $\bigcup_{\zeta^{\prime}} S_{\zeta^{\prime}}^{(D)}$ for all $\zeta^{\prime}$ belonging to the common part of $C-E$ and $\left|z-z_{0}\right| \leqq r$. We denote by $\rho_{1}$ the distance of $M_{r}$ from $w_{0}$. Let $\rho_{2}$ be a positive number such that $\left|f(z)-w_{0}\right| \geqq \dot{\rho}_{2}>0$ on $K \cdot D$. We denote by $\rho$ a positive number less than $\min \left(\rho_{1}, \rho_{2}\right)$. Since $w_{0}$ is a cluster value of $w=f(z)$ at $z_{0}$, there exists a sequence of points $z_{\mu}(\mu=1,2, \ldots)$ inside $(K) \cdot D$, $(K)$ denoting the interior of $K$, tending to $z_{0}$ such that $w_{\mu}=f\left(z_{\mu}\right)$ tends to $w_{0}$.

10) R. Nevanlinna: 1. c. 1), pages 132 and 134.

11) K. Noshiro: On the theory of the cluster sets of analytic functions, Journ. Fac. of Sci., Hokkaido Imp. Univ. 6 (1938), pp. 217-231; Cf. theorem 4. 
We keep hereafter the sequence $z_{\mu}(\mu=1,2, \ldots)$ fixed. Consider the open set $D$ of points $z$ inside $(K) \cdot D$ whose images $w=f(z)$ lie in $(c):\left|w-w_{0}\right|<0$. Then $D_{0}$ consists of a finite or an enumerable number of connected domains $d_{0}$ Denote by $A_{\mu}$ the component containing $z_{\mu}$; some $A_{\mu}$ may coincide with one other.

First we consider the case in which there are infinitely many distinct com. ponents $A_{\mu}$. For the sake of simplicity, we suppose that $\Delta_{k} \neq A_{\nu}$ if $\mu \neq \nu$. Then, we easily show that $\Delta_{1}(\mu=1,2, \ldots)$ converges to $z_{0}$. For, if otherwise there exists a circle $K^{\prime}:\left|z-z_{0}\right|=r^{\prime}(<r)$ such that $K^{\prime} \cdot E=0$ and $K^{\prime} \cdot \Delta_{\mu_{n}} \neq 0(n=1$, $2, \ldots)$, where $A_{\mu_{n}}$ denotes a sub-sequence of $A_{\mu}$. Let $\zeta_{n}$ be any boundary point of $A_{\mu_{3}}$, lying on the circle $K^{\prime}$ and $\zeta_{0}$ be a point of accumulation of the sequence $\zeta_{n}(n=1,2, \ldots)$. Since $f\left(\zeta_{n}\right)$ lies on the circle $c:\left|w-w_{0}\right|=\rho, \zeta_{0}$ must belong to either $C-E$ or $D$. However, either of two cases leads to a contradiction, because either the set $M_{r}$ intersects the circle $\left|w-w_{0}\right|=\rho$ or infinitely many niveau curves: $\left|f(z)-w_{0}\right|=\rho$ intersect any neighbourhood of $\zeta_{0}$, while $w=f(z)$ is pseudo-regular $(A)$ in $D$. If $A_{\mu}$ is compact in $D$, then it is evident that $w$ $=f(z)$ takes every value in $(c):\left|w-w_{v}\right|<\rho$. If $\Delta_{\mu}$ is not compact in $D$, its boundary consists of a closed subset $E_{\mu}$ of $E$ and a finite or an enumerable number of analytic curves inside $D$; by Lemma 1, the value-set $\mathfrak{D}_{\mu}$ of $w=f(z)$ in $A_{\mu}$ is everywhere dense in $(c):\left|w-w_{0}\right|<\rho$, what is the same, the closure $\overline{\mathfrak{D}}_{\mu}$ coincides with $\left|w-w_{0}\right| \leqq \rho$. Considering that $\Delta_{\mu}(\mu=1,2, \ldots)$ converges to $z_{0}$, we see that the cluster set $S_{z_{0}}^{(D)}$ includes the closed circular disc $\left|w-w_{0}\right|$ $\leqq \rho$ 。

Next, let $r_{n}$ and $\rho_{n}$ be two decreasing sequences of positive numbers tending to zero, such that, for each $n, r_{n}$ and $\rho_{n}$ are selected as stated above, and consider two sequences of circles $K_{n}:\left|z-z_{0}\right|=r_{n}$ and $c_{n}:\left|w-w_{0}\right|=\rho_{n} \quad(n=1,2$, ...). Denote by $\Delta_{\mu}^{(n)}$ the component with an interior point $z_{\mu}$, which is an inverse image of $\left(c_{n}\right):\left|w-w_{0}\right|<\rho_{n}$. If the sequence $\Delta_{\mu}^{(n)}\left(\mu \geqslant N_{k}\right)$ consists of infinitely many distinct domains for at least one $n$, then the reasoning used above shows that $S_{z_{0}}^{(D)}$ includes the closed disc $\left|w-w_{0}\right| \leqq \rho_{n}$. Thus, we have only to consider the case in which the sequence $A_{\mu}^{(n)}$ consists of only a finite number of distinct dornains for every $n$. Denote by $\Delta_{1}$ any $A_{\mu}^{(1)}$ containing a sub-sequence $\left\{z_{k^{2}}^{(1)}\right\}$ of $\left\{z_{\mu}\right\}$, and by $A_{2}$ any $A_{\mu}^{(2)}$ containing a sub-sequence $\left\{z_{\mu}^{(i)}\right\}$ of $\left\{z_{\mu}^{(1)}\right\}$ and so on. Thus, we obtain a new sequence of domains $\left\{\Delta_{n}\right\}$ such that $A_{1} \supset A_{2} \supset \ldots \supset A_{n} \supset \ldots$ and each $A_{n}$ has a boundary point $z_{n}$ in common. Accordingly, since the value-set of $w=f(z)$ in $A_{n}$ is included in $\left(c_{n}\right):\left|w-w_{0}\right|$ $<\rho_{n}$ and the diameter of $\Delta_{n}$ tends to zero as $n \rightarrow \infty$, there exists an asymptotic path $A$ of $w=f(z)$ reaching $z_{0}$ along which $w=f(z)$ converges to $w_{0}$. Denote 
by $\Omega_{0}$ the component containing $w_{0}$ of the complementary set of $S_{z_{0}}^{*(c)}$ with respect to the $w$-plane. We shall now show that $w=f(z)$ takes every value, except two possible exceptions, belonging to $\Omega_{0}$ infinitely often in any neighbourhood of $z_{0}$. Without loss of generality, we may suppose that $\Omega_{0}$ does not contain $w$ $=\infty$. Suppose, contrary to the assertion, that there are three exceptional values $w_{1}, w_{2}, w_{3}$ in $\Omega_{0}$. Then, there exists a positive number $\eta_{1}$ such that $f(z)$ $\neq w_{1}, w_{2}, w_{3}$ in the common part of $D$ and $U\left(z_{0}, \eta_{1}\right):\left|z-z_{0}\right|<\eta_{1}$. Inside $\Omega_{0}$ we draw a simple closed regular analytic curve $\Gamma$ which surrounds $w_{0}, w_{1}, w_{2}$ and passes through $w_{3}$, and whose interior consists only of interior points of $\Omega_{0}$. By hypothesis, we can select a positive number $\eta\left(<\eta_{1}\right)$, arbitrarily small, such that, $K^{\prime}$ denoting the circle $\left|z-z_{0}\right|=\eta, K^{\prime} \cdot(C-E)=0$ and the closure $M_{\eta}$ of the union $\bigcup_{\zeta^{\prime}} S_{\zeta^{\prime}}^{\left(D^{\prime}\right)}$ for all $\zeta^{\prime}$ belonging to the common part of $C-E$ and $\left|z-z_{0}\right| \leqq \eta$ lies outside $\Gamma$. We may assume that the image of $A$ by $w=f(z)$ is a curve lying completely in the interior of $\Gamma$. Consider the set $D_{\eta}$ of points $z$ inside the intersection of $D$ and $U\left(z_{0}, \eta\right)$ such that $w=f(z)$ lies in the interior of $\Gamma$. Then the open set $D_{\eta}$ consists of at most an enumerable number of connected components. We shall denote by $\Delta$ the component which contains the asymptotic path $\Lambda$. It is easily seen that the boundary of $\Delta$ consists of a finite number of arcs of the circle $K^{\prime}$, a finite or an enumerable number of analytic contours inside $D$ and a closed subset $E_{0}$ of $E_{\text {. }}$ Further it should be noticed that $\Delta$ is simply connected. For, by hypothesis, $E$ is included in a single boundary-component $C_{0}$ of the boundary $C$ of $D$ and the frontier of $\Delta$ contains no closed analytic contour, since every analytic contour of $\Delta$ is transformed by $w=f(z)$ into a curve lying on the simple closed curve $\Gamma$ passing through an exceptional value $w_{3}$. Denote by $\Phi$ the Riemannian image of $\Delta$ transformed by $w=f(z)$ in a one-one manner and by $\Phi_{0}$ the domain obtained by excluding two points $w_{1}$ and $w_{2}$ from the interior of $\Gamma$. Then, $\emptyset$ is a simply connected covering surface of basic surface $\emptyset_{0}$ whose Euler's characteristic is equal to 1 . With an aid of Evans' theorem stated before, we can prove, without difficulty, that $\emptyset$ satisfies the condition of regular exhaustion (with a slightly modified form) in Ahlfors' sense. But this will lead to a contradiction by Ahlfors' main theorem on covering surfaces. ${ }^{12)}$ Thus, it is proved that $S_{z_{0}}^{(D)}-S_{z_{0}}^{*(C)}$ is an open set.

Suppose that the open set $\Omega=S_{z_{0}}^{(D)}-S_{z_{0}}^{*(C)}$ is not empty. Let $\Omega_{n}$ be any connected component of $\Omega$. We shall now prove that $w=f(z)$ takes every value, with two possible exceptions, belonging to $\Omega_{n}$ infinitely often in any neighbourhood of $z_{0}$. We may suppose that $\Omega_{n}$ does not contain $w=\infty$. Contrary to the

12) L. Ahlfors: Zur Theorie der Überlagerungsflächen, Acta Math. 65 (1935), pp. 157-194.

R. Nevanlinna: 1. c. 1), Cf. p. 323. K. Noshiro: 1. c. 8). 
assertion, we suppose that there are three exceptional values $w_{0}, w_{1}$ and $w_{\mathrm{a}}$ in $2_{n}$. Then, there exists a positive number $\eta_{1}$ such that $f(z) \neq w_{0}, w_{1}, w_{2}$ in the common part of $D$ and $U\left(z_{0}, \eta_{1}\right):\left|z-z_{0}\right|<\eta_{1}$. Inside $Q_{n}$ we draw a simple closed regular analytic curve $\Gamma$ which surrounds $w_{0}, w_{1}$ and passes through $w_{\text {s }}$, and whose interior consists only of interior points of $\Omega_{n}$. We can select a positive number $\eta\left(\left\langle\eta_{1}\right)\right.$, arbitrarily small, such that, $K^{\prime}$ denoting the circle $\left|z-z_{0}\right|=\eta, K^{\prime} \cdot(C-E)=0$ and the closure $M_{\eta}$ of the union $\bigcup_{\zeta^{\prime}} S_{\zeta^{\prime}}^{\left.()^{\prime}\right)}$ for all $\zeta^{\prime}$ belonging to the common part of $C-E$ and $\left|z-z_{0}\right| \leqq \eta$ lies outside $\Gamma$. Now, by Lemma 2 either $w_{0}$ is an asymptotic value of $w=f(z)$ at $z_{3}$ or there exists a sequence of $\zeta_{i z}$ in $E$ tending to $z_{0}$ such that $w_{0}$ is an asymptotic value at each $\zeta_{n}$. Consequently it is possible to find a point $\zeta_{0}$ (distinct from $z_{0}$ or not) belonging to $E \cdot U\left(z_{0}, \eta\right)$ such that $w_{0}$ is an asymptotic value of $w=f(z)$ at $\varepsilon_{0}$. Let $A$ be the asymptotic path with the asyrnptotic value $w_{0}$ at $\sigma_{0}$. We may assume that the image of $\Lambda$ by $w=f(z)$ is a curve lying completely inside $\Gamma$. Consider the set $D_{\eta}$ of points $z$ inside the intersection of $D$ and $U\left(z_{0}, \eta\right)$ such that $w=f(z)$ lies inside $I$. Now, we denote by $A$ the component, of $D_{\eta}$, which contains the asymptotic path $A$. Since $A$ must be simply connected, we would arrive at a contradiction. ${ }^{13)}$

Mathematical Institute, Nagoya University

13) K. Noshiro: 1. c. 8). 